\title{
MAPK Pathway in Skeletal Muscle Diseases
}

\section{Hannah W.S. Geisler, Hao Shi ${ }^{*}$ and David E. Gerrard}

Department of Animal and Poultry Sciences, Virginia Polytechnic Institute and State University, USA

${ }^{*}$ Corresponding author: Hao Shi, Department of Animal and Poultry Sciences, Virginia Polytechnic Institute and State University, 304 Litton-Reaves Hall, Blacksburg, VA 24061, USA, Fax: (540) 231-3010, Tel: (540) 231-9663, E-mail: haoshi@vt.edu

Citation: Hannah W.S. Geisler, Hao Shi, David E. Gerrard (2013) MAP Kinase Pathway in Skeletal Muscle Diseases. J Vet Sci Anim Husb 1(1): e104. doi: 10.15744/2348-9790.1.e104

Received Date: July 08, 2013 Accepted Date: July 29, 2013 Published Date: August 01, 2013

\begin{abstract}
Mitogen-Activated Protein Kinase (MAPK) pathway is a signal transduction pathway that functions in a wide range of physiological and pathophysiological cellular events including cell proliferation, differentiation, apoptosis, migration, inflammation, metabolic disorders and diseases. In skeletal muscle, it plays an essential role in muscle fiber specialization, muscle mass maintenance, damage induced muscle regeneration and muscle diseases. This review provides an overview of MAPK pathway and its pathophysiological role in skeletal muscle diseases with a primary focus on muscular dystrophy and atrophy.
\end{abstract}

\section{MAPK Pathway}

MAPK pathway consists of at least 4 subfamilies that include extracellular signal-regulated kinase 1 and 2 (ERK 1/2), p38 $\alpha / \beta / \gamma / \delta$ MAPK, c-Jun NH2-terminal kinases 1,2 , and 3 (JNK 1/2/3), and ERK5 [1-4]. MAPKs are a family of protein phosphorylating enzymes [5] that regulate diverse aspects of cellular responses in physiology, immunology, neurobiology, and energy metabolism [6]. Cellular activities regulated by MAPKs include proliferation, differentiation, apoptosis, motility $[7,8]$, stress responses, inflammation, and innate immunity [9-11]. MAPK functions through transcriptional activation and posttranslational modification on the downstream substrates. Specifically, MAPKs are involved in the production of antimicrobial factors, cytokines, chemokines, and other inflammatory mediatory factors [12]. In the central nervous system, MAPKs are required for proper neuronal axonal development [13]. MAPKs also play a critical part in energy metabolism through modulating lipid metabolism [14-16] and skeletal muscle growth and fiber type [6]. Given the fact that MAPKs play such a fundamental and integral role in a broad range of biological processes, interference of this pathway and their downstream effector proteins may have detrimental consequences leading to either metabolic disorder or diseases. This review mainly focuses on the functional role of MAPK pathway in skeletal muscle diseases.

MAPKs phosphorylate serine and threonine residues on their substrates [8] which may include protein kinases, phospholipids, transcription factors and even cytoskeletal proteins [17]. MAPKs are activated through a three-tiered phosphorylation relay that transmits signals from the cell surface into biochemical responses: MAPKs are phosphorylated and thus activated by upstream Mitogen-Activated Protein Kinase Kinases (MKKs) which are phosphorylated and activated by their upstream MKK kinases (MKKKs) [18,19]. Given the fact that each tier of phosphorylation consists of multiple kinases, one may question how an extracellular signal is decoded and transduced into a specific cellular response in a temporal and spatial manner. The complexity of regulation of the MAPKs provides a platform on which diverse signals converge and are precisely deciphered to generate a specific signal that fine-tunes the signaling network within a cell. On the other hand, an activated MAPK signal has to be curtailed in a timely manner so that cell will not overreact to a stimulus so to maintain metabolic homeostasis. One mechanism involves the dephosphorylation and thus inactivation of MAPKs by the MAPK phosphatases (MKPs) [5]. MKPs, also known as dual-specificity protein phosphatases (DUSPs), are a group of 10 catalytically active protein tyrosine phosphatases [9,20,21]. MKPs inactivate MAPKs through dephosphorylation of MAPKs on regulatory threonine and tyrosine residues. Though the substrates of the MKPs are to some extent overlapping, MKPs achieve their substrate specificity through binding affinity and sub-cellular localization [22].

\section{MAPKs and Muscular Dystrophy}

Muscular dystrophy (MD) is a group of over 30 genetic diseases that cause degeneration of skeletal muscles during voluntary movement and overall progressive weakness in muscle strength [23]. These diseases can be classified into nine major types including Duchenne, Becker, Myotonic, Congenital, Emery-Dreifuss, Facioscapulohumeral (FSHD), Limb-girdle, 
Distal, and Oculopharyngeal. The most common and severe muscular dystrophy is Duchenne muscular dystrophy (DMD) which accounts for greater than $50 \%$ of all disease cases. DMD is a prevalent muscular dystrophy in humans, affecting 1 : 3,500 males in the United States [24]. Naturally occurring or spontaneous mutations in the DMD gene has been reported in Canine breeds such as Golden Retriever [25], German ShortHaired Pointer [26], and Cavalier King Charles[27]. Canine studies of X-linked DMD revealed that pups from an affected male were stunted on growth and showed progressive weakness and gait abnormalities which lead to muscle atrophy more over mine over they also exhibited fibrosis and contractures by 6 months of age. Smaller breeds are less affected than large crosses [28]. In feline DMD models, the disorder is referred to as hypertrophic feline muscular dystrophy (HFMD) due to a loss of dystrophin in skeletal and heart muscles, skeletal muscle hypertrophy occurs especially in the tongue muscles [29].

The role of MAPKs in dystrophy is not completely understood, and the inconclusive evidence from different diseases and experimental settings confounds a definitive role of MAPKs in the pathogenesis of muscular dystrophy [22]. Interestingly, a mechanistic link has been built between the dystrophin-glycoprotein complex (DGC), a structure that is dysfunctional in muscular dystrophy and the MAPK pathway. The DGC not only provides structural support to cells, but also bridges extracellular stimuli and intracellular reaction through specific physical interactions with intracellular proteins [30]. Some of these proteins, such as adapter protein Grb2, are involved in the MAPK signal transduction, which suggests that DGC may function to transduce signals through the MAPKs to maintain skeletal muscle fiber viability [22]. Our recent work on MAPK phosphatases (MKPs) has added another layer of regulation of MAPKs in Duchenne muscular dystrophy. MKP-1 is a nuclear phosphatase that dephosphrylates p38 MAPK, JNK, and ERK1/2 not only in skeletal muscle but also in the immune, metabolic, and nervous system as well [9,31-34]. In the cardiotoxin-induced skeletal muscle regeneration, knockout of MKP-1 impaired the recovery of the damaged muscle likely through the effect of p38 MAPK pathways on muscle stem cell function [33], since the precocious differentiation of muscle stem cells caused by enhanced p38 MAPK may slow down the injured muscle from returning to a normal musculature [33]. Interestingly, when another MKP family member MKP-5 was knocked out, muscle regeneration following damage was greatly improved [35]. When MKP-5 was knocked out in the $m d x$ mouse, an animal model for Duchenne muscular dystrophy, the dystrophinopathy was ameliorated and muscle functions were restored [35]. The obvious discrepancy between MKP-1 and MKP-5 knockout studies can be explained by the fact that MKP-5 is both nuclear and cytosolic, though its substrates include p38 MAPK, JNK and ERK1/2, it functions on distinct pools of MAPKs from MKP-1 due to their subcellular localization [35]. Together, these findings suggest that MAPKs may play pivotal roles in the phathogenesis of muscular dystrophy, however, the definitive role of each MAPK in the network of signaling pathways that contribute to the progression of the diseases remains to be further investigated.

\section{MAPKs and Muscle Atrophy}

Skeletal muscle atrophy is characterized by decrease in cell size through organelle, cytoplasm, and protein loss [36]. Biochemically, muscle atrophy is a result of negative balance between protein synthesis and degradation. This imbalance in protein turnover occurs during fasting, disuse of selected skeletal muscles, and a plethora of systemic diseases such as cancer, diabetes mellitus, AIDS, sepsis, and Cushing's Syndrome [37].

Traditionally, it was thought there are two types of muscular atrophy in skeletal muscles: neurogenic atrophy and muscle atrophy through ubiquitin-dependent proteolysis [38]. Healthy muscle maintains its size, structure, and function through innvervation of motor neurons to skeletal muscle fibers, and through balance of protein accretion. Upon disruption of the nervous system, denervation occurs and neurogenic muscle atrophy ensues [38]. Such disorders are seen in amyotrophic lateral sclerosis (ALS), Guillain-Barré syndrome, polio, and polyneuropathy which ultimately leads to paralysis [38]. In contrast, ubiquitin-dependent proteolysis occurs during increased rate of protein degradation through the ubiquitin-proteasome pathway [39] and transcriptional adaptations called "atrophy program" [40]. Recently, emerging evidence indicates that there is another equally important protein breakdown machinery in skeletal muscle called autophagy-lysosome system. In mammals, there are three different types: macroautophagy, chaperone-mediated autophagy (CMA), and microautophagy [41], though the mechanistic aspect of each category remains yet to be further defined.

Due to the fact that muscle atrophy occurs in a large number of diseases and disorders in both humans and animals, it is a focal point of interest in basic research and clinical investigation. In agriculture and veterinary medicine, muscle atrophy caused by malnutrition is the culprit of inefficiently growing animals and alters meat quality. Atrophy, along with caloric restriction, induces cytoskeletal remodeling of muscle fibers which may contribute to lower quality less tender meat product [42]. Aging also proves to be a factor for muscle atrophy. In the hindlimbs of older dogs, a growing insensitivity to adenosine triphosphate (ATP) occurs and muscle fibers undergo fibrosis which weakens muscle [43].

Among the various pathways that participate in the pathogenesis of muscle atrophy, MAPKs play a critical role. There are several lines of evidence that support this notion. First, when MKP-1, a phosphatase that dephosphorylates and thus inactivate MAPKs, was overexpressed in skeletal muscle fibers, it induced profound muscle fiber atrophy possibly through the ubiquitin-proteasome pathways [44]. This atrophic effect may be conferred through ERK1/2 signaling pathway, yet it does not exclude the possibility of a combined effect of ERK, JNK, and p38 MAPK pathways [44]. Second, given the fact that MAPKs play integral roles in innate immunity [45], it is reasonable to speculate that MAPK-mediated cytokine secretion may serve as an indirect effect on skeletal muscle atrophy as seen in chronic inflammation diseases. Specifically, activation of JNK and p38 MAPKs are involved in cytokine-induced 
muscle degeneration. ERK1/2 pathway, however, counteracts muscle wasting through enhanced protein synthesis by its control of ribosomal RNA gene expression [46]. Finally, diverse regimens of exercises have been developed to counter age induced skeletal muscle atrophy in humans and animals. Analysis of exercise with aged animals has revealed that concentric activity, which includes weight training, may slow the weakening of muscles [47]. This type of exercise is not commonly employed in companion animal rehabilitation but water resistance activities may improve the quality of life in elderly animals [43]. Analysis of the MAPK activation during different exercise protocols revealed that ERK1/2 phosphorylation increases in nearly all forms of exercises, whereas p38 MAPK and JNK are activated in specific exercise protocols [48-51]. Together, these findings demonstrate that MAPK signal transduction pathway may serve as a potential therapeutic target to combat skeletal muscle atrophy in human and animals.

\section{Future Perspectives}

A wealth of data supports a critical role of the MAPKs in the pathogenesis of skeletal muscle diseases. MAPKs are activated and inactivated in a temporal and spatial manner through their upstream kinases and a family of phosphatases to achieve specific biological responses. Research in this area has begun to shed a light on the complexity and hierarchy of the MAPK regulation and how this signaling pathway is integrated into the intertwined signaling network system within a cell. Knockout animal models targeting specific MAPKs and their isoforms are useful tools to define the role of each individual MAP kinase in a specific muscle disease. Although mice are an excellent model to study the mechanistic insight of the MAPK in muscle diseases, other animal models are valuable especially in veterinary medicine and agriculture. Large animals may be more challenging for gene manipulation, yet the work in pigs has brought promise for the use of large animals to study human diseases. Animals with spontaneous mutations in genes of interest are another valuable tool to study muscle diseases. In all, research in the MAPK pathway will undoubtedly broaden our understanding of the etiology and pathogenesis of muscle disease and the molecular signatures that define the pathological process. All of these efforts are targeted to develop therapeutic means to improve the health of humans and animals. Given the fact that MAPK signaling pathway is ubiquitous in all cell types, drugs that inhibit MAPK signaling may have undesired effects. As such, identification of the downstream effectors of the MAPKs that convey MAPK effects in a specific disease will be an optimal choice.

\section{References}

1. Bogoyevitch MA (2006) The isoform-specific functions of the c-Jun Nterminal Kinases (JNKs): differences revealed by gene targeting. Bioessays 28 : 923-934.
2. Bogoyevitch MA, Court NW (2004) Counting on mitogen-activated protein kinases--ERKs 3, 4, 5, 6, 7 and 8. Cell Signal 16: 1345-1354.

3. Whitmarsh AJ (2006) The JIP family of MAPK scaffold proteins. Biochem Soc Trans 34: 828-832.

4. Zarubin T, Han J (2005) Activation and signaling of the p38 MAP kinase pathway. Cell Res 15: 11-18.

5. Lawan A, Shi H, Gatzke F, Bennett AM (2013) Diversity and specificity of the mitogen-activated protein kinase phosphatase-1 functions. Cell Mol Life Sci 70: 223-237.

6. Wancket LM, Frazier WJ, Liu Y (2012) Mitogen-activated protein kinase phosphatase (MKP)-1 in immunology, physiology, and disease. Life Sci 90: 237-248.

7. Cuevas BD, Abell AN, Johnson GL (2007) Role of mitogen-activated protein kinase kinase kinases in signal integration. Oncogene 26: 3159-3171.

8. Pearson G, Robinson F, Beers Gibson T, Xu BE, Karandikar M, et al. (2001) Mitogen-activated protein (MAP) kinase pathways: regulation and physiological functions. Endocr Rev 22: 153-183.

9. Boutros T, Chevet E, Metrakos P (2008) Mitogen-activated protein (MAP) kinase/MAP kinase phosphatase regulation: roles in cell growth, death, and cancer. Pharmacol Rev 60: 261-310.

10. Duan W, Wong WS (2006) Targeting mitogen-activated protein kinases for asthma. Curr Drug Targets 7: 691-698.

11. Jeffrey KL, Camps M, Rommel C, Mackay CR (2007) Targeting dualspecificity phosphatases: manipulating MAP kinase signalling and immune responses. Nat Rev Drug Discov 6: 391-403.

12. Dong C, Davis RJ, Flavell RA (2002) MAP kinases in the immune response. Annu Rev Immunol 20: 55-72.

13. Jeanneteau F, Deinhardt K, Miyoshi G, Bennett AM, Chao MV (2010) The MAP kinase phosphatase MKP-1 regulates BDNF-induced axon branching. Nat Neurosci 13: 1373-1379.

14. Flach RJ, Qin H, Zhang L, Bennett AM (2011) Loss of mitogen-activated protein kinase phosphatase-1 protects from hepatic steatosis by repression of cell death-inducing DNA fragmentation factor A (DFFA)-like effector C (CIDEC)/fat-specific protein 27. J Biol Chem 286: 22195-22202.

15. Roth RJ, Le AM, Zhang L, Kahn M, Samuel VT, et al. (2009) MAPK phosphatase-1 facilitates the loss of oxidative myofibers associated with obesity in mice. J Clin Invest 119: 3817-3829.

16. Wu JJ, Roth RJ, Anderson EJ, Hong EG, Lee MK, et al. (2006) Mice lacking MAP kinase phosphatase- 1 have enhanced MAP kinase activity and resistance to diet-induced obesity. Cell Metab 4: 61-73.

17. Johnson GL, Lapadat R (2002) Mitogen-activated protein kinase pathways mediated by ERK, JNK, and p38 protein kinases. Science 298: 1911-1912.

18. Dhanasekaran DN, Kashef K, Lee CM, Xu H, Reddy EP (2007) Scaffold proteins of MAP-kinase modules. Oncogene 26: 3185-3202.

19. Turjanski AG, Vaque JP, Gutkind JS (2007) MAP kinases and the control of nuclear events. Oncogene 26: 3240-3253.

20. Soulsby M, Bennett AM (2009) Physiological signaling specificity by protein tyrosine phosphatases. Physiology 24: 281-289.

21. Tonks NK (2006) Protein tyrosine phosphatases: from genes, to function, to disease. Nat Rev Mol Cell Biol 7: 833-846.

22. Shi H, Bennett AM (2012) Mitogen-Activated Protein Kinases and Mitogen-Activated Protein Kinase Phosphatases in Regenerative Myogenesis and Muscular Dystrophy. In: Madhuri Hegde (eds) Muscular Dystrophy, Winchester, United Kingdom.

23. (2011) (NINDS) NIoNDaS Muscular dystrophy: Hope through research. 
24. Porter JD (2000) Introduction to muscular dystrophy. Microsc Res Tech 48: $127-130$.

25. Cooper BJ, Winand NJ, Stedman H, Valentine BA, Hoffman EP, et al. (1988) The homologue of the Duchenne locus is defective in X-linked muscular dystrophy of dogs. Nature 334: 154-156.

26. Schatzberg SJ, Olby NJ, Breen M, Anderson LV, Langford CF, et al. (1999) Molecular analysis of a spontaneous dystrophin 'knockout' dog. Neuromuscul Disord 9: 289-295.

27. Walmsley GL, Arechavala-Gomeza V, Fernandez-Fuente M, Burke MM Nagel N, et al. (2010) A duchenne muscular dystrophy gene hot spot mutation in dystrophin-deficient cavalier king charles spaniels is amenable to exon 51 skipping. PLoS One 5: e8647.

28. Valentine BA, Cooper BJ, de Lahunta A, O'Quinn R, Blue JT (1988) Canine X-linked muscular dystrophy. An animal model of Duchenne muscular dystrophy: clinical studies. J Neurol Sci 88: 69-81.

29. Shelton GD, Engvall E (2005) Canine and feline models of human inherited muscle diseases. Neuromuscul Disord 15: 127-138.

30. Rando TA (2001) The dystrophin-glycoprotein complex, cellular signaling, and the regulation of cell survival in the muscular dystrophies. Muscle Nerve 24: 1575-1594.

31. Flach RJ, Bennett AM (2010) MAP kinase phosphatase-1--a new player at the nexus between sarcopenia and metabolic disease. Aging (Albany NY) 2: $170-176$.

32. Roth Flach RJ, Bennett AM (2010) Mitogen-activated protein kinase phosphatase-1- a potential therapeutic target in metabolic disease. Expert Opin Ther Targets 14: 1323-1332.

33. Shi H, Boadu E, Mercan F, Le AM, Flach RJ, et al. (2010) MAP kinase phosphatase-1 deficiency impairs skeletal muscle regeneration and exacerbates muscular dystrophy. FASEB J 24: 2985-2997.

34. Wu JJ, Bennett AM (2005) Essential role for mitogen-activated protein (MAP) kinase phosphatase-1 in stress-responsive MAP kinase and cell survival signaling. J Biol Chem 280: 16461-16466.

35. Shi H, Verma M, Zhang L, Dong C, Flavell RA, et al. (2013) Improved regenerative myogenesis and muscular dystrophy in mice lacking Mkp5. J Clin Invest 123: 2064-2077.

36. Sandri M (2008) Signaling in muscle atrophy and hypertrophy. Physiology 23: $160-170$.

37. Lecker SH, Solomon V, Mitch WE, Goldberg AL (1999) Muscle protein breakdown and the critical role of the ubiquitin-proteasome pathway in normal and disease states. J Nutr 129: 227S-237S.

38. Moresi V, Williams AH, Meadows E, Flynn JM, Potthoff MJ, et al. (2010) Myogenin and class II HDACs control neurogenic muscle atrophy by inducing E3 ubiquitin ligases. Cell 143: 35-45.
39. Jagoe RT, Goldberg AL (2001) What do we really know about the ubiquitin-proteasome pathway in muscle atrophy? Curr Opin Clin Nutr Metab Care 4: 183-190.

40. Jagoe RT, Lecker SH, Gomes M, Goldberg AL (2002) Patterns of gene expression in atrophying skeletal muscles: response to food deprivation. FASEB J 16: 1697-1712.

41. Bonaldo P, Sandri M (2013) Cellular and molecular mechanisms of muscle atrophy. Dis Model Mech 6: 25-39.

42. Byrne KA, Wang YH, Lehnert SA, Harper GS, McWilliam SM, et al (2005) Gene expression profiling of muscle tissue in Brahman steers during nutritional restriction. J Anim Sci 83: 1-12.

43. Wakshlag JJ (2010) Dietary protein consumption in the healthy aging companion animal. Proceedings of the Nestle Purina Companion Anima Nutrition Summit: Focus on Gerontology 2010.

44. Shi H, Scheffler JM, Zeng C, Pleitner JM, Hannon KM, et al. (2009) Mitogen-activated protein kinase signaling is necessary for the maintenance of skeletal muscle mass. American journal of physiology Cell physiology 296: C1040-C1048.

45. Chi H, Barry SP, Roth RJ, Wu JJ, Jones EA, et al. (2006) Dynamic regulation of pro- and anti-inflammatory cytokines by MAPK phosphatase 1 (MKP-1) in innate immune responses. Proc Natl Acad Sci U S A 103: 22742279.

46. Stefanovsky V, Langlois F, Gagnon-Kugler T, Rothblum LI, Moss T (2006) Growth factor signaling regulates elongation of RNA polymerase I transcription in mammals via UBF phosphorylation and $\mathrm{r}$-chromatin remodeling. Molecular cell 21: 629-639.

47. Sakamoto K, Goodyear LJ (2002) Invited review: intracellular signaling in contracting skeletal muscle. J Appl Physiol 93: 369-383.

48. Goodyear LJ, Chang PY, Sherwood DJ, Dufresne SD, Moller DE (1996) Effects of exercise and insulin on mitogen-activated protein kinase signaling pathways in rat skeletal muscle. Am J Physiol 271: E403-E408.

49. Martineau LC, Gardiner PF (2001) Insight into skeletal muscle mechanotransduction: MAPK activation is quantitatively related to tension. J App Physiol 91: 693-702.

50. Nader GA, Esser KA (2001) Intracellular signaling specificity in skeletal muscle in response to different modes of exercise. J Appl Physiol 90: 19361942.

51. Ryder JW, Fahlman R, Wallberg-Henriksson H, Alessi DR, Krook A, et al (2000) Effect of contraction on mitogen-activated protein kinase signal transduction in skeletal muscle. Involvement of the mitogen- and stress-activated protein kinase 1. J Biol Chem 275: 1457-1462.

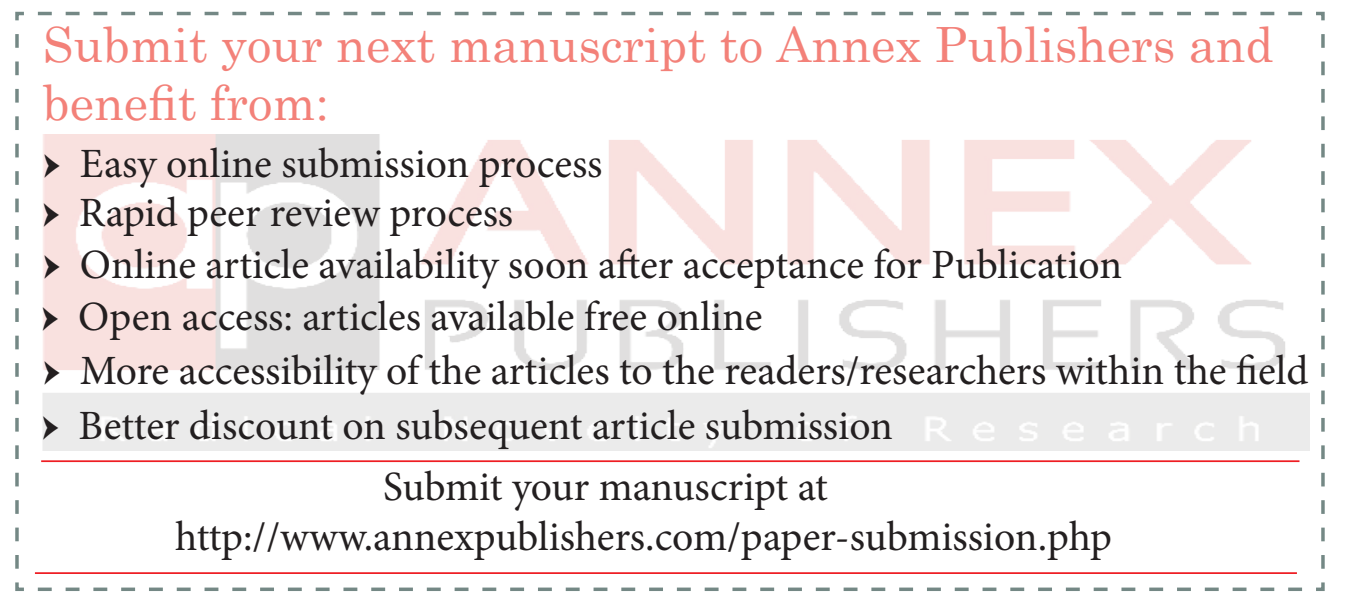

